\title{
Inference-Driven Dynamic Access Scheme for Interference Management in Heterogeneous Networks
}

\author{
Ahmed R. Elsherif, Zhi Ding, Xin Liu, Jyri Hämäläinen, and Risto Wichman \\ University of California, Davis, California 95616 \\ Aalto University, Espoo, Finland \\ \{arelsherif,zding,xinliu\}@ucdavis.edu \\ \{jyri.hamalainen, risto.wichman\}@aalto.fi
}

\begin{abstract}
We propose a scheme for interference management in wireless heterogeneous networks. We, specifically, consider the problem of downlink interference of a Home eNB (HeNB) to nearby macrocell user equipments (MUEs) served by a macrocell base station. We propose a distributed, self-learning channel access mechanism that enables the HeNB to adaptively change its access to channels shared with neighboring MUEs. Basically, the HeNB accesses the channel with a probability that is updated based on the overheard uplink feedback information from neighboring MUEs. We formulate an optimization problem with the goal of maximizing the achieved rate for the HeNB under the constraint of maintaining a quality of service (QoS) requirement for neighboring MUEs.
\end{abstract}

\section{INTRODUCTION}

Recent statistics have shown that more than $50 \%$ of voice calls and more than $70 \%$ of data traffic originate from indoor cellular subscribers [1]. Yet, historically, substantial activities in wireless research and development have focused on high speed mobile users and the resulting fast fading channel problems. In fact, current cellular technologies and deployment tend to exhibit poor indoor coverage, especially for high speed data services whose broadband requirement suffers severe channel distortions and packet losses in complex indoor environment. One recent proposal for improving indoor wireless coverage introduces the promising concept of heterogeneous networking (HetNet) and more specifically the deployment of femtocells [2]. A femtocell is an indoor cellular base station that connects subscribers at a high speed and low power by reusing the same cellular spectrum. Femtocell base stations connect with the core service network by utilizing broadband connection such as Digital Subscriber Line (DSL), cable modem, or an RF as a backhaul channel.

From the network operator's point of view, femtocells improve indoor coverage, and offload traffic from the macrocell, which helps improve the macrocell throughput and link reliability. Moreover, the cost of a femtocell, including equipment and deployment, is much lower than that of a macrocell base station deployed by the operator. The concept of heterogeneous networking, and femtocells in particular, has been already proposed in the standardization process for next generation communication systems such as LTE(-A) and WiMAX [3].

This material is based on works supported by the National Science Foundation under Grant CNS1147930
Femtocell base stations are referred to as Home eNBs (HeNBs) in LTE standardization. Because the backhaul connection of HeNB to the core-network relies on commercial Internet, HeNB control channels and data traffics cannot be fully coordinated by the mobile network controller. In fact, HeNB can receive control information of its neighboring macrocell basestation (MBS). But such information undergoes Internet delays and cannot be relied upon for HeNB resource assignment and interference control.

HetNet infrastructure has been studied, in terms of handoff, resource management, and capacity analysis [4]-[5]. In such studies, spectrum allocation between macro- and micro-cell has been rather static and the traffic has been voice-only. In contrast, HetNet is a natural evolution from the rigid cell coverage to a more flexible and better radio coverage in response to better information with respective to environment variability, user mobility, and data traffic patterns. Cooperative HetNet operation centers on the resource allocation and channel access of multiple networks simultaneously to achieve maximum throughput and high utility of available spectrum.

Cooperative HetNet can be viewed as a more general and intelligent form of cognitive networking. In the literature, the concept of cognitive radio typically refers to radio units having the ability to sense the environment and to adapt its frequency, power, and transmission schemes [6]. In the cooperative HetNet context, the macrocell user equipment (MUE) is considered the primary user (PU) that has to have rate assurance, whereas the home user equipment (HUE) acts as the secondary user (SU) in cognitive radio scenarios. There is a significant amount of work done on this context. Dynamic access strategies based on sensing outcomes are one of the common techniques on literature to solve such problems. In [7] the PU is not aware of the SU activity. SU can access any available channel but it can not access any channel occupied by the PU. Problems are solved and characterized using a twodimensional Markov chain model.

Various mechanisms have been considered for femtocell resource management, including downlink power control, e.g., in [8], MIMO beamforming though precode matrix index selection [9], and interference avoidance by overhearing MUE resource block allocation information [10] and by using measurement/distance-based power control [11]. Femtocell 
interference management can be considered as a special case of HetNet resource management. In [12], the authors proposed an adaptive power control technique to limit the transmission power of femtocells in order to maximize frame utilization. One major drawback of this scheme [12] is that it is oblivious to the QoS need of the MUE that experiences the HeNB interference.

Several works have applied feedback information to improve SU performance. In [13] the SU is using the ACK/NAK received from the PU to estimate the Markov chain that the PU is following. In [14], an SU actively transmits probing signals to observe the changing transmission power of the PU in response. In [15], a wideband OFDM cognitive radio dynamically changes its subcarrier usage based on the average power and transmission probability of the narrow-band PU devices. Our principal idea is to overhear receiver feedback signals at different network nodes to facilitate cooperative operation in HetNet. In [16] and [17], we proposed a resource allocation scheme that uses the overheard feedback information from the MUEs together with downlink control information (DCI) to estimate the likelihood that a resource block is unoccupied, assigned to a nearby MUE (indoor), or assigned to a far enough MUE (outdoor).

In [18], the authors considered the uplink interference mitigation problem between HUEs and neighboring MUEs. They proposed a stochastic resource allocation strategy that tries to roughly separate the spectrum usage in a probabilistic way between HUEs and indoor MUEs. Therefore, the cross-tier interference from macrocell to femtocell is most likely coming from outdoor MUEs that are behind attenuating building structures. The drawback of this scheme is that HeNB and MBS would need to correctly classify MUEs as indoor versus outdoor user equipments. More importantly, the given access probabilities are fixed and not adaptive to channel conditions. In our work, we consider an adaptive policy that updates the access probability based on the feedback information received from the MUE. Basically, we use the ACK/NAK feedback signals together with CQI information to dynamically change the HUE access probabilities for the physical resource block (PRB) clusters. These feedback information are available in most recent cellular systems such as LTE(-A) and WiMAX.

The rest of the paper is organized as follows : In Section II, we describe the system model that will be employed in the rest of the paper. In Section III, we present the problem formulation and our proposed solution. In Section IV, we highlight some practical issues for the implementation of the proposed scheme, while Section V gives the numerical evaluation of the proposed solution. Finally, Section VI concludes the paper.

\section{SYSTEM MODEL}

We consider a HetNet that consists of an HeNB deployed within the coverage of a MBS. The HeNB has $N_{h}$ HUEs whereas the MBS has $N_{m}$ MUEs. Because of spectrumsharing between HUEs and MUEs, the downlink mutual interference between HeNBs and MUEs is captured as an Xchannel model as shown in Fig. 1 (for the simple case of one
MBS, one HeNB, one MUE, and one HUE). The X-channel model means that the transmission from the HeNB to the HUE causes interference to the MUE, and MBS transmission causes interference to the downlink reception at the HUE.

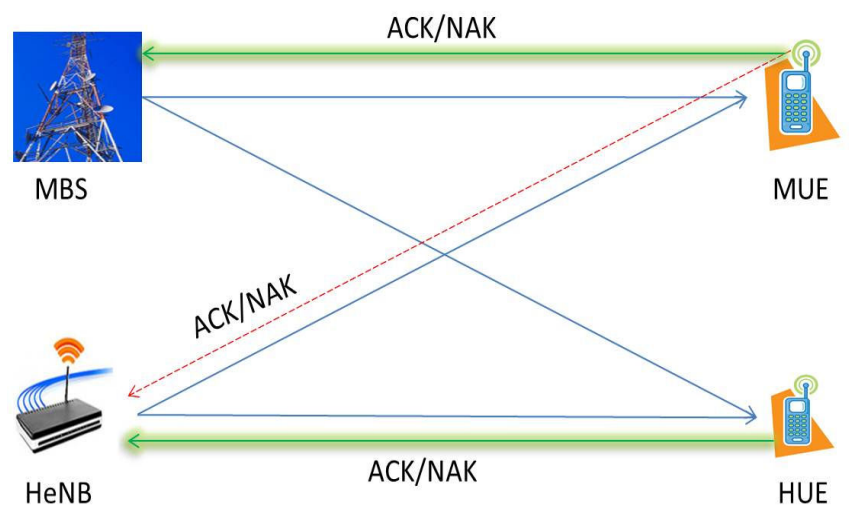

Fig. 1: Downlink interference channel model.

The MBS resource allocation is based on its own primary network information, including its MUE CQI reports, traffic load, H-ARQ, and buffer status. On the other hand, the HeNB resource allocation must account for interference management to reduce interference impact on MUEs. However, because MBS resource allocation is not coordinated with the HeNB, the HeNB assignment of downlink resources to its HUE must be flexible and reactive. The HeNB should assign resources that are rarely used by the MUE's downlink such that there will be fewer interfering opportunities between the downlink signals for MUE and HUE.

\section{PRoblem Formulation}

In this paper, we focus on distributed approaches to HetNet cooperation, which is complementary to high level centralized approaches. In particular, centralized approaches to resource allocation and interference management can be effective and appropriate for large time-scale cooperations, but our investigation on distributed interference management targets highly dynamic techniques to respond to rapid, small time-scale network reconfiguration and adaptivity needs. We first consider the problem formulation for one MBS and one HeNB and consider the generalization in Section IV.

The allocation of the MBS is dynamic and varies from frame to frame. For this reason, HeNB resource allocation cannot wait for MBS assignment first because of the lack of response time. Practically, we would like to design a selflearning resource allocation policy for the HeNB for better interference management by reducing the interference and probability of PRB collision (i.e. probability that both MBS and $\mathrm{HeNB}$ use the same PRB/resource for transmission to their UEs). The HeNB must allocate its resources by considering interference caused by its downlink transmission to the MUEs, especially those who are close enough to the HeNB.

Since MUEs are configured to transmit periodically and ondemand channel quality indication (CQI) reports for specific 
PRB clusters, such channel information is utilized by MBS in its PRB assignment to ensure good connectivity. By configuring the HeNB, as well, to receive CQI reports from both MUEs and HUEs, the HeNB can perform downlink PRBs assignment to reduce the potential for PRB collision and to provide better link quality.

The MBS must also consider its overall available resources and its subscribers' requests. Nevertheless, in reaction to a high quality CQI report from an MUE for a given PRB cluster $i$, the MBS MAC should assign this PRB cluster for the MUE downlink with higher probability. In other words, the probability $p_{i}$ of assigning PRB cluster $i$ to the MUE should be such that $p_{i} \geq p_{j}$ if the CQI value of cluster $i$ is higher than that of $j$, i.e., $\mathrm{CQI}_{m}(i) \geq \mathrm{CQI}_{m}(j)$. In response, it would, then, be more reasonable for the HeNB to assign cluster $i$ to an HUE with lower probability of $q_{i} \leq q_{j}$. Hence, if PRB clusters $i$ and $j$ have CQI levels of $\mathrm{CQI}_{m}(i)$ and $\mathrm{CQI}_{m}(j)$, respectively, with quality order $\mathrm{CQI}_{m}(i) \geq \mathrm{CQI}_{m}(j)$, then the HeNB should assign its HUE for downlink with probability $q_{i} \leq q_{j}$.

In many applications, QoS guarantee at the MUE is essential when admitting HUE services. To ensure QoS for the MUE downlink, we define 2 MUE states for each channel PRB cluster $i$ to be estimated for each MUE within interference range. During downlink resource allocation for each MUE within interference range, the HeNB should estimate two states, namely, the probability of access and the SINR of MUE at time $k:\left[\hat{p}_{i}^{(k)}, \widehat{\operatorname{SINR}}_{m}^{(k)}(i)\right]$. As will be shown in Section IV, the HeNB can use its overheard CQI report, along with ACK/NAK feedbacks to update the state estimates for each PRB cluster $i$.

Given the estimated state information, the HeNB can determine the average data rate of MUE downlink as a function of the HUE downlink access probability $\left\{q_{i}\right\}$ as follows

$$
R_{m}\left(\left\{q_{i}\right\}\right)=\mathbb{E}\left\{\sum_{i} \log _{2}\left(1+\left(\operatorname{SINR}_{m}(i) \mid F(i), M(i)\right)\right)\right\}
$$

where $\widehat{\operatorname{SINR}}_{m}(i)$ is the SINR of the MUE on cluster $i$ and $F(i)$ and $M(i)$ are indicator functions for accessing cluster $i$ for HUE and MUE, respectively, as follows

$$
F(i)= \begin{cases}1 & \text { HUE will access cluster } i \\ 0 & \text { otherwise, }\end{cases}
$$

and

$$
M(i)= \begin{cases}1 & \text { MUE will access cluster } i \\ 0 & \text { otherwise. }\end{cases}
$$

The expectation in (1) is on the different values of $F(i)$ and $H(i)$. The probabilities of the $F(i)$ and $H(i)$ are given as follows

$$
\begin{aligned}
\operatorname{Pr}(F(i)=1) & =q_{i} \\
\operatorname{Pr}(F(i)=0) & =1-q_{i} \\
\operatorname{Pr}(M(i)=1) & =p_{i} \\
\operatorname{Pr}(M(i)=0) & =1-p_{i}
\end{aligned}
$$

The value of $\operatorname{Pr}(M(i)=1)$ is estimated at the HeNB as $\hat{p}_{i}$. Therefore, the estimate of the average data rate of MUE at the HeNB can be written as

$$
\begin{aligned}
R_{m}\left(\left\{q_{i}\right\}\right)=\sum_{i}\left\{\hat{p}_{i}\left(1-q_{i}\right) \log _{2}\left(1+\widehat{\operatorname{SINR}}_{m}(i)\right)\right. \\
\left.+\hat{p}_{i} q_{i} \log _{2}\left(1+\operatorname{SINR}_{m f}(i)\right)\right\}
\end{aligned}
$$

where $\widehat{\operatorname{SINR}}_{m}(i)$ is the HeNB estimate of the MUE's SNR that can be obtained based on its overheard CQI reports. The HeNB also has to estimate the MUE's SINR during collision with HUE transmission using the same PRB cluster denoted by $\operatorname{SINR}_{m f}(i)$. To estimate $\operatorname{SINR}_{m f}(i)$, the HeNB needs to determine the channel path-loss $\left\|H_{10}\right\|^{2}$ between itself and MUE based on MUE signal transmission and/or reciprocity. Let the background noise spectrum $N_{0}$ be identical at all nodes, we have,

$$
\operatorname{SINR}_{m f}(i) \approx \frac{\operatorname{SINR}_{m}(i) \cdot N_{0}}{N_{0}+P_{f}(i)\left\|H_{10, i}\right\|^{2}},
$$

where $P_{f}(i)$ is the HeNB transmission power over PRB cluster $i$.

Given its own $\operatorname{SINR}_{f}(i)$ based on CQI reports from HUEs, the HeNB can determine the probabilistic resource allocation parameters through the following linear programming (LP) optimization

$$
\begin{array}{ll}
\max _{\left\{q_{i}\right\}} & \sum_{i} q_{i} \log _{2}\left(1+\operatorname{SINR}_{f}(i)\right), \\
\text { s. t. } & R_{m}\left(\left\{q_{i}\right\}\right)>R_{\text {min }} \\
& 0 \leq q_{i} \leq 1 \\
& \sum_{i} q_{i} \leq s_{q} \text {,max. }
\end{array}
$$

The objective function in (7a) is to maximize the sum achievable rate by the HUEs over all channel clusters. The constraint in (7b) is a QoS constraint for the MUE where a minimum rate $R_{\min }$ has to be guaranteed for the MUE. Constrain (7c) determines the range for the HUE access probability $q_{i}$. The constraint in (7d) sets an upper limit, $s_{q, \max }$, on the sum of the access probabilities over all clusters. The parameter $s_{q}$, max can be varied to change the priorities of channel access of the HeNB and MBS. Because this optimization is essentially a linear programming problem, it can be solved efficiently using simplex or interior point methods.

\section{MBS Downlink Allocation Probability Estimate}

So far, we have assumed that the probability of MUE downlink occupancy of a certain cluster, $p_{i}$, is known at the HeNB. However, this probability should be estimated at the HeNB. To get the estimate, $\hat{p}_{i}$, efficiently and accurately, the HeNB should first have access to the basic policies of the MBS downlink assignment. Nevertheless, because the HeNB can only acquire partial RLC control and feedback information, it cannot fully anticipate the MBS downlink assignment for each PRB cluster. Therefore, our estimate should rely on dynamic 
algorithms that utilize HeNB observations, including MBS downlink history, MBS data rate, MUE CQI reports, MUE and HUE packet ACK/NAK, HeNB power, and estimated pathloss.

One possible simple estimate for the MBS downlink allocation probability at HeNB can be obtained based on DLC information observation up to time $k-1$ as follows

$\hat{p}_{i}(k)=\operatorname{Prob}[$ channel $i$ assigned to $\operatorname{MUE} \mid \operatorname{DLC}(0: k-1)]$.

The HeNB is assumed to receive only local information that includes interactive control signals between the MBS and MUE, but does not have access to the global primary network information and reports. Based on its detection of the MBS downlink frames, the HeNB can estimate a probability of PRB allocation $p_{i}$. Further CQI reports can be used by the HeNB to update its estimate of probability $p_{i}$ via

$$
\hat{p}_{i}(k+1)=\alpha_{m} \hat{p}_{i}(k)+\left(1-\alpha_{m}\right) \frac{\mathrm{CQI}_{m}(i)}{\sum_{j} \mathrm{CQI}_{m}(j)},
$$

where parameter $\alpha_{m}$ is the forgetting factor that can be adjusted based on the variability rate of the MUE reports. In a static case, $\alpha_{m}=1$, whereas for rapidly varying channels, $\alpha_{m}=0$.

\section{IMPLEMENTATION ISSUES}

In this section, we discuss some of the issues that need to be considered for practical implementation of our proposed scheme.

\section{A. MBS Downlink Channel State Estimate}

By developing accurate and timely estimate of the interference channel $\widehat{\operatorname{SINR}}_{m}(i)$, it becomes possible for the HeNB to assess the effect of its own downlink interference during PRB collision with MBS downlink. Thus, we should develop a good SINR estimate for MUEs that fall within the HeNB interference range. Because the HeNB action will affect the SINR of the MUE, we plan to develop a partially observable Markov decision process (POMDP) for HeNB to allocate and to observe MUE and HUE feedback reports (including CQI, NAK/ACK) for SINR estimation.

\section{B. Downlink Resource Allocation of Multiple HeNBs}

When multiple HeNBs must allocate resources to their UEs without centralized coordination, they represent independent downlink interference to every MUE. We must tackle the critical issue of joint resource allocation without significantly lowering the MUE downlink rate and without causing substantial collisions of resource usage among the multiple downlink connections. Since the severity of multiple HeNB interferences can be reflected from the MUE reports of CQI and ACK/NAK feedback, we will investigate how each HeNB can effectively utilize the MUE's ACK/NAK response and CQI reports for channel state estimation. We also plan to develop PRB allocation policies for multiple HeNB downlink without collectively aggravating the MUE downlink throughput. One solution is that each HeNB ignores the possible presence of other HeNBs and applies the same optimization problem as in (7a) and (7b). This will lead to an aggressive approach as each HeNB considers itself the only source of interference and uses (6) to get an estimate of MUE's SINR under collision, which is, in fact, lower than what the reality might be due to other HeNBs' interference.

\section{Downlink Resource Allocation with Multiple MUE Feed-} backs

We will also investigate the more general problem for scenarios in which the HeNB downlink may interfere with multiple MUE nodes during downlink. Based on the optimization framework, the rate constraint of every MUE will be taken into account by expanding (7b) into multiple constraints. From the feedback from multiple MUE nodes, the MUE CQI reports allow us to estimate the PRB access probability (8) for the linear programming optimization of HeNB downlink resource allocation.

\section{Numerical Evaluation}

In this section, we provide simulation results for the proposed dynamic access strategy in comparison with the fixed access strategy in [18]. The system consists of a HeNB deployed within the coverage of a MBS. The HeNB is serving a number of HUEs and is assumed able to overhear the uplink feedback reports of neighboring MUEs connected to the MBS. We assume that the HeNB has closed access and, thus, a nearby MUE will not be allowed to access the HeNB. In our simulation results, we consider only one MUE in the coverage range of the HeNB. The simulation parameters are summarized in Table I.

\begin{tabular}{|c|c|}
\hline Parameter & Value \\
\hline Number of clusters & 8 \\
\hline Channel & Multipath, Fast fading \\
\hline MUE/HUE Noise Figure & $7 \mathrm{~dB}$ \\
\hline MBS Transmit Power & $20 \mathrm{dBm}$ \\
\hline HeNB Transmit Power & $151.1+42.8 * \log _{10}\left(d_{0} / 1000\right)$ \\
\hline MBS to MUE Path Loss & $d_{0}:$ distance between HeNB \& HUE \\
\hline HeNB to HUE Path Loss & $\begin{array}{c}127+30 * \log _{10}\left(d_{1} / 1000\right) \\
d_{1}: \text { distance between MBS \& MUE }\end{array}$ \\
\hline
\end{tabular}

TABLE I: Simulation Parameters.

Fig. 2 shows the achieved HUE and MUE rates for our proposed scheme as well as for the fixed access scheme as a function of the distance between the HeNB and the neighboring MUE, $d_{10}$. The minimum rate requirement of the MUE in this simulation is 5 Mbps. We consider 8 clusters where a cluster can correspond to a resource block group (RBG), in LTE terminology, or any number of RBGs. For the fixed access probability case we consider equi-probable access of all clusters. Fig. 2 shows that our proposed scheme gives higher sum HUE rate compared to the fixed access case. Moreover, for $s_{q, \max }=1$, the proposed scheme also achieves higher MUE rate compared to the fixed access scheme. However, for higher values of $s_{q}$,max, Fig. 2 shows that the fixed access case achieves an MUE rate which is higher than the sufficient 
minimum rate requirement of the MUE. The proposed scheme also achieves a value that is higher than the minimum rate requirement of the MUE, although lower than that for the fixed case, however, it has more flexibility to achieve higher HUE rate compared to the fixed access strategy.
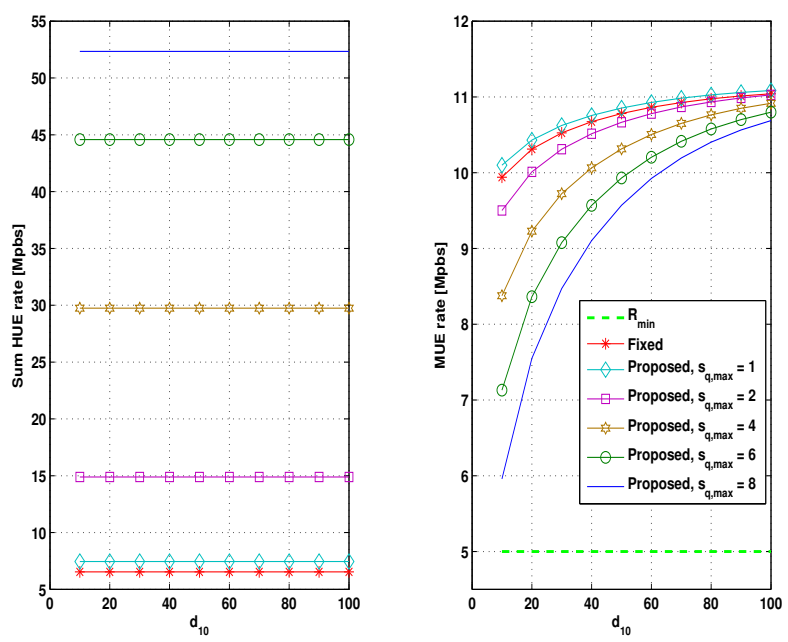

Fig. 2: Sum HUE / MUE rate versus $d_{10}$.

Moreover, from Fig. 2, we notice that as the value of $s_{q, \text { max }}$ increases, the proposed scheme achieves higher rates for the HUEs, while still meeting the MUE's minimum rate requirement. Increasing the value of $s_{q}$,max means that, on average, we allow the HUE to access more clusters and, thus, it can achieve higher rate.

Fig. 3 shows the total achieved rate of both the MUE and the HUEs. It is clear that even for $s_{q, \max }=1$, our proposed schemes achieves higher sum rate compared to the fixed access probability case. As the value of $s_{q}$,max increases, the proposed scheme achieves higher sum rate.

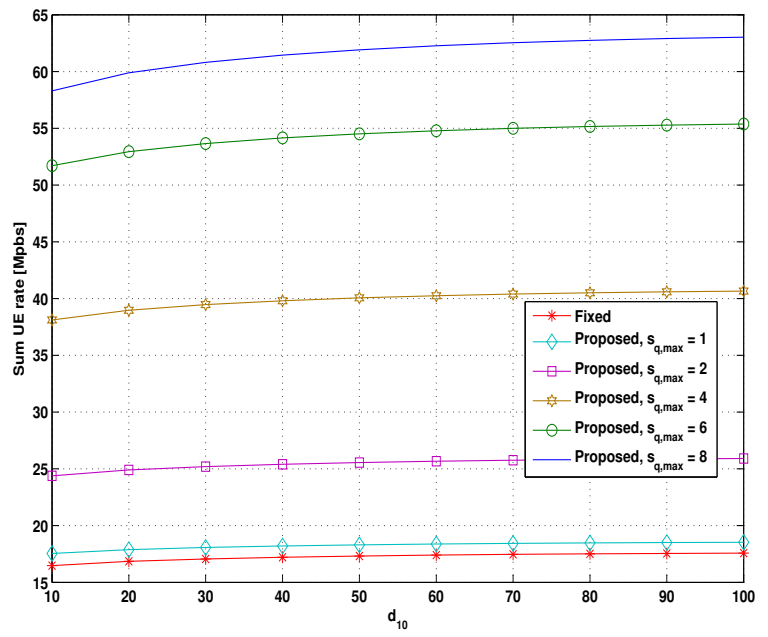

Fig. 3: Total UE rate versus $d_{10}$.
On the other hand, in Fig. 4, we fix the distance between the HeNB and the MUE, $d_{10}=100 \mathrm{~m}$, and evaluate the performance for different values of MUE's minimum rate requirement, $R_{\min }$. Fig. 4 shows that the proposed scheme achieves the minimum rate requirement of the MUE for the considered range of $R_{\min }$ and also achieves a higher HUE rate compared to the fixed access strategy.
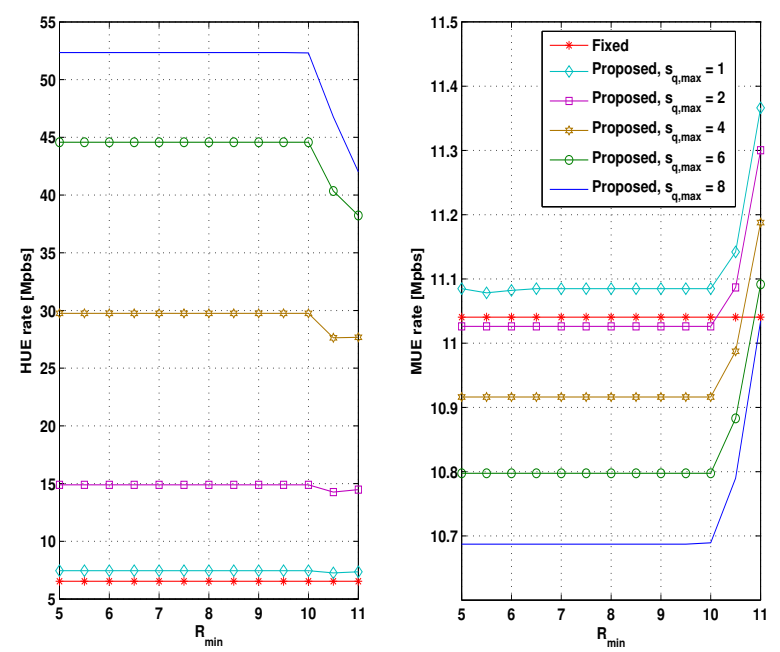

Fig. 4: Sum HUE / MUE rate versus $R_{\min }$.

Fig. 5 shows the corresponding total UE rate versus $R_{\text {min }}$. It is clear that the proposed dynamic access scheme achieves higher total UE rate than the fixed access strategy for all values of $s_{q, \max }$.

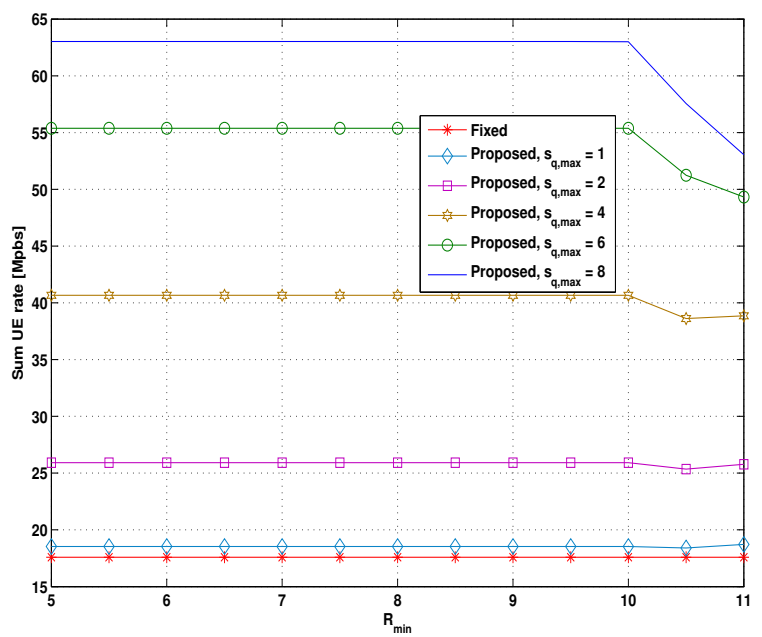

Fig. 5: Total UE rate versus $R_{\text {min }}$.

\section{CONCLUSION}

We developed a priority-based, distributed, and self-learning channel access strategy that enables adaptive HeNB access 
of shared channels resulting in low interference levels to neighboring MUEs and, hence, low packet failure rates. Our basic idea is to allow the HeNB to use the overheard uplink feedback information from neighboring MUEs to update the HUE access probability for different channels. We formulated an optimization problem with the objective of maximizing the achieved throughput by the HeNB while meeting QoS constraints for neighboring MUEs. We showed that this optimization problem is a linear programming problem that can be solved easily and efficiently. We also show, through numerical evaluations, that our proposed dynamic access scheme performs better than a corresponding fixed access scheme. We also highlight and discuss some implementation issues that we need to consider in future work for a more practical implementation of our solution.

\section{REFERENCES}

[1] Presentations by ABI Research, Picochip, Arivana, IP access, Gartner, Telefonica Espana, 2nd Int'l Conf. Home Access Points and Femtocells. [Online]. Available: http://www.avrenevents.com/dallas-femto2007/ purchase_presentations.htm

[2] V. Chandrasekhar, J. Andrews, and A. Gatherer, "Femtocell Networks: a Survey," IEEE Communications Magazine, 46(9):59-67, 2008.

[3] J. Zhang and G. de la Roche, Femtocells : Technologies and Deployment, JohnWiley \& Sons, New York, NY, USA, 2010.

[4] K.L Yeung and S. Nanda, "Optimal Mobile-Determined Micro-Macro Cell Selection," in IEEE PIMRC, 1995.

[5] Li-Chun Wang, G.L. Stuber, and Chin-Tau Lea, "Architecture Design, Frequency Planning, and Performance Analysis for a Microcell/Macrocell Overlaying System," in IEEE Transactions on Vehicular Technology, 1997.

[6] I. F. Akyildiz, W. Y. Lee, M. C. Vuran, and S. Mohanty, "NeXt generation / dynamic spectrum access / cognitive radio wireless networks: A survey," Computer Networks Journal (Elsevier), Sept. 2006.
[7] Y. Zhang, "Dynamic Spectrum Access in Cognitive Radio Wireless Networks," in IEEE International Conference on Communications, Dec. 2008 : 4927-4932.

[8] X. Li, L. Qian, and D. Kataria, "Downlink Power Control in Cochannel Macrocell Femtocell Overlay," 2009 43rd Annual Conference on Information Sciences and Systems, pages 383-388, 2009.

[9] C. Jiang, L. J. Cimini, and N. Himayat, "MIMO Mode Adaptation in Femtocellular Systems," Computer Engineering, pages 122-127, 2011.

[10] M. Sahin, I. Guvenc, M. Jeong, and H. Arslan, "Handling CCI and ICI in OFDMA Femtocell Networks Through Frequency Scheduling," IEEE Trans. on Consumer Electronics, 55(4):1936-1944, 2009.

[11] H. Claussen, L. T. W. Ho, and L. G Samuel, "Self-Optimization of Coverage for Femtocell Deployments," 2008 Wireless Telecomunications Symposium, pages 278-285, 2008.

[12] P. Mach, Z. Becvar, "Dynamic Power Control Mechanism for Femtocells Based on the Frame Utilization," in 6th International Conference Wireless and Mobile Communications, 2010.

[13] Q. Zhao, L. Tong, A. Swami and Y. Chen, "Decentralized cognitive MAC for opportunistic spectrum access in ad hoc networks: A POMDP framework," IEEE Journal on Selected Areas in Communications 25.3 (2007) : 589-600.

[14] R. Zhang and Y. C. Liang, "Exploiting Hidden Power Feedbacks in Cognitive Radio Networks," 3rd IEEE Intl. Symp. on New Frontiers in Dynamic Spectrum Access Networks, 2008.

[15] H. Rahul, N. Kushman, D. Katabi, C. Sodini, and F. Edalat, "Learning to Share: Narrowband-Friendly Wideband Networks," SIGCOMM Comput. Commun. Rev., 38(4):147-158, 2008.

[16] A. R. Elsherif, Z. Ding, X. Liu, J. Hämäläinen, and R. Wichman, "Shadow Chasing : A Resource Allocation Scheme For Heterogeneous Networks," in 7th International Conference on Cognitive Radio Oriented Wireless Networks (Crowncom), 2012.

[17] A. R. Elsherif, Z. Ding, X. Liu, J. Hämäläinen, "Shadow Chasing Enhancement in Resource Allocation For Heterogeneous Networks," in IEEE Global Communication Conference (Globecom), 2012.

[18] Z. Zheng, J. Hämäläinen, and Y. Yang, "On Uplink Power Control Optimization and Distributed Resource Allocation in Femtocell Networks," in VTC Spring 2011 BeFEMTO workshop, 15 May, (Budapest, Hungary), 2011 